\title{
Minimisation of Generation Variability of a Group of Wind Plants
}

\author{
Dubravko Sabolic ${ }^{* 1}$, Alan Župan ${ }^{2}$, Roman Malaric ${ }^{3}$ \\ ${ }^{1}$ Croatian Transmission System Operator Ltd., Kupska 4, Zagreb, Croatia \\ e-mail: dubravko.sabolic@ @ops.hr \\ ${ }^{2}$ Croatian Transmission System Operator Ltd., Kupska 4, Zagreb, Croatia \\ e-mail: alan.zupan@ @ops.hr \\ ${ }^{3}$ Department of Electrical Engineering Fundamentals and Measurements, Faculty of Electrical Engineering \\ and Computing, University of Zagreb, Unska 2, Zagreb, Croatia \\ e-mail: roman.malaric@fer.hr
}

Cite as: Sabolić, D., Župan, A., Malarić, R., Minimisation of Generation Variability of a Group of Wind Plants, J. sustain. dev. energy water environ. syst., 5(3), pp 466-479, 2017, DOI: http://dx.doi.org/10.13044/j.sdewes.d5.0157

\begin{abstract}
Minimisation of variability of energy delivered from a group of wind plants into the power system using portfolio theory approach was studied. One of the assumptions of that theory is Gaussian distribution of the sample, which is not satisfied in case of wind generation. Therefore, optimisation of a "portfolio" of plants with different goal functions was studied. It was supposed that a decision on distribution of a fixed amount of generation capacity to be installed among a set of geographical locations with known wind statistics is to be made with minimised variability of generation as a goal. In that way the statistical cancellation of variability would be used in the best possible manner. This article is a brief report on results of such an investigation. An example of nine locations in Croatia was used. These locations' wind statistics are known from historic generation data.
\end{abstract}

\section{KEYWORDS}

Wind plants, Variability of generation, Portfolio theory, Non-Gaussian distribution, Optimisation, Statistical cancellation.

\section{INTRODUCTION}

Optimisation of wind plant operation regarding temporal variability of generation with a portfolio theory approach was studied in past (see e.g., [1] or [2]). It is important to use available geographical locations for placement of wind plants in an optimal way regarding total variability of generation, because in that way one best utilizes the "natural potential" for statistical cancellation of variability. This variability incurs additional costs in the value chain of production and delivery of electrical energy. The system costs of Renewable Sources (RES) were presented in [3].

It is worth mentioning that problems with variability of RES such as wind or solar is routinely used in "political arena" as an argument against proliferation of RES's. Also, a number of researchers point to various types of problems related to short term variability, be it purely technical/operational ones, or the ones related to economic side of the system operation. There are numerous research articles dealing with "integration costs", the

\footnotetext{
* Corresponding author
} 
externalities incurred to the power system by variable renewables. Such studies aim at estimation of the costs that need to be added upon Life-time Costs Of Electricity (LCOE) from variable renewable sources, to yield a figure called "system Life-time Costs Of Electricity" (sLCOE, [3]), that is supposed to be a better metric of RES life-cycle cost. However, they do not aim at social welfare analyses of RES integration.

Gross et al. [4] analyse impacts and costs of intermittent generation on the British grid, and conclude that there is no evidence of decreased reliability, although the power system operation faces more challenges. Milligan and Kirby [5] aim to separate economic value of energy from costs and calculate wind integration costs as the difference between total costs with and without wind generation, and conclude that the economic value of wind-generated energy is somewhat lower than base load. Smith et al. [6] argue that wind integration costs of up to USD 5 to USD 6/MWh of wind energy can be expected for capacity penetrations of up to $20 \%$ to $30 \%$ of peak load. Holttinen et al. [7] build upon best practices from wind integration studies and conclude, among others, that system operation and functioning electricity markets at less than day-ahead time scales help reduce forecast errors of wind power. DeCesaro and Porter [8] find wind integration costs at $20 \%$ penetration level to be about one tenth of the wholesale value of energy. GE Energy [9] finds that both variability, and uncertainty, of wind production impact grid operation. However, uncertainty appears to be a bigger challenge.

Another type of research papers studies the marginal economic value of energy produced, to conclude that increase in RES penetration in overall energy mix decreases economic value of variable renewables. For instance, Fripp and Wiser [10] find that the potential difference in wholesale market value between better correlated and poorly correlated wind sites is modest, on the order of 5-10 percent. Borenstein [11] gives an interesting study on another type of variable renewable generation (solar), pointing out that institutional setting in which capacity costs are recovered through a flat rate per-kWh fee, so that the wholesale prices are much less volatile, is not favourable to economic value of generated energy. Lamont [12] develops a theoretical framework to find an expression for the marginal value of an intermittent technology as a function of the average system marginal cost, the capacity factor of the generator, and the covariance between the generator's hourly production and the hourly system marginal cost. Mills and Wiser [13] provide a very detailed study of economic value of variable generation at high penetration levels and decompose relevant integration costs, concluding, among others, that solar-generated energy has high value at low penetrtation rates, whereas wind energy can exceed solar and concentrating solar power in value by high penetrations. Nicolosi [14] stresses that market organization aspects can have significant impact on value of electricity generated from variable renewable sources. Hirth [15] argues that, from the standpoint of overall social welfare, the optimal penetration rate of RES occurs where increasing sLCOE curve, and decreasing curve of marginal economic value of energy, intersect.

There is no doubt that it is in the best interest of RES industry, the power sector, and the society as a whole, to avoid all unnecessary costs. Therefore, utilising all possible methods to reduce variability can be important not only from the perspective of increased Transmission System Operator's (TSO's) costs and operational risks, but from the policy and politics point of view. Leaving the variability unmanaged can be regarded as irrationality because it incurs more costs and technical problems, without a need to do so. If the costs related to balancing of variability were monotonically increasing with the variability itself (which is a reasonable proposition), minimization of variability would mean minimization of variability-associated costs. The first-line beneficiaries of it would naturally be TSOs. They could then, for example, transfer the gain to the consumers by lowering transmission tariff, or employ the surplus to serve more variable RES in their 
networks, or employ it for any other useful purpose, rather than spill it for something that could have been managed by purely organizational (i.e., virtually costless) means.

As it comes to the investors in variable RES, their interest in lowering the variability depends on market design. If for example the wind plant operators were exposed to balancing costs (like "conventional" generators are), decrease of variability would immediately provide financial benefits. By contrast, if the RES were isolated from short-term market signals, the investors would still have interest in decrease of total variability as the grid would be able to accept more installed capacity, everything else unchanged. Thus, there would be more investment opportunities. On the other hand, more installed wind plant capacities means more competition, more energy generated with (almost) zero variable cost, and thus, lower economic value of energy, which is generally not in the investor's interest. Overall social welfare (that is, the sum of net benefits to producers, consumers, and TSOs) undoubtedly increases with optimization of variability because it leads to elimination of a part of total costs of electricity, without increasing any other cost.

One possible approach to minimisation of variability is using the portfolio theory, formulated first by Markowitz [16], whose theory brought the 1990 Nobel prize in economics to its founder, but also received a good measure of criticism (see e.g., in Elton and Gruber [17]). One of the most notable problems with it is the one-period assumption, that is, the assumption that future statistical properties of varying financial instruments would remain the same as in the past period of observed time-series data. While it really can be a problem in the financial world, because market conditions, as well as economic conditions of institutions that issued the securities involved, can indeed change quite abruptly in short periods of time, it may not be of so much importance in case of wind speeds because the behaviour of winds and other meteorological phenomena exhibits certain periodicity over longer time spans. Thus, knowing the historical data of wind speeds at a certain location, which can be easily converted into data on energy generation per unit of installed capacity, may allow for a trustworthy portfolio analysis. The second major objection on Markowitz's theory pertains to assumption of Gaussian distribution of observed data population. There is a lot of evidence that wind production is not at all Gaussian (see for example Sabolić [18]). In Monahan et al. [19] probability density function of land surface wind speeds is characterized using a global network of observations, to conclude that it is essentially a Weibull distribution, which is skewed (non-symmetric about expected value). Ouarda et al. [20] provide comparative analysis of goodness-of-fit of different theoretical statistical distributions to actual measured wind speed data, to conclude that the two-parameter Weibull model performs the best.

\section{Methods}

One of the most important goals of research presented in this article was establishing whether the portfolio theory, as a conceptual tool, is any good for optimising portfolios of wind plants. Our working hypothesis is that the portfolio theory can in principle be used as a tool for minimization of generation variability.

To investigate this, we carried out a simulation on 2014 measured generation data in 15 minute intervals at nine locations of wind plants in Croatia. It is important to stress that the results of the analyses "have really nothing to do with these power plants themselves". The measurements from them were only used to calculate, in the first step, the percentage of the plant's output relative to the amount of energy that could have been produced if a plant operated at full installed capacity during a given 15 minute interval. The time series obtained in that way were used as a starting point for portfolio analysis.

In this investigation only the generation variability was used as parameter to minimise. In reality, there are other costs related to locations of wind plants, so the future research 
should include these parameters, as well. However, it was outside the intended scope of this paper.

Say now that one wants to allocate a fixed amount of new generation capacity, $C$, to install in $N$ available locations with known wind statistics during a certain period of time. For the sake of simplicity, let $C=1$ (that is, the measurement unit for electrical power does not matter). The basic form of portfolio theory optimisation problem can then be stated as follows:

$$
\min \sigma_{p}^{2}=\Sigma_{i} \Sigma_{j} r_{i} r_{j} \sigma_{i} \sigma_{j} \rho_{i j} \quad i, j \in[1, N]
$$

$$
\begin{aligned}
& \text { subject to constraints: } \sum_{i} r_{i}=1 \\
& r_{i} \in[0,1] \\
& E_{p}=\Sigma_{i} r_{i} E_{i} \\
& i \in[1, N] \\
& \forall i \in[1, N] \\
& i \in[1, N]
\end{aligned}
$$

where $r_{i}$ is a weight factor, a share in total installed capacity allocated to the location $i$, $E_{i}$ is expected hourly production expressed as a percentage of capacity allocated to the location $i, \sigma_{i}$ is the standard deviation of production (defined in the above described manner) in percentage points, $\rho_{i j}$ is the correlation coefficient between time series of production at locations $i$ and $j, E_{p}$ is expected total hourly production of the portfolio, also expressed in percentages of total installed capacity (thus $E_{p}$ is the load factor of the portfolio), and $\sigma_{p}^{2}$ is portfolio's variance. The first constraint states that all the weight factors must add up to 1 (that is, actual installation capacities must add up to $C$ ). The second constraint means that a wind plant cannot be installed with negative capacity. The third constraint is simply a definition of expected load factor of a portfolio of wind plants. The definition of portfolio's variance, $\sigma_{p}^{2}$, is given in the problem statement line, because it is the function to be minimised.

In the research presented here, analytical solutions without the second constraint were also calculated using standard methods (Merton [21]; Lando and Poulsen [22]), because they form a hyperbolic "efficient frontier" in $\sigma-E$ plane (the loci of points with the lowest possible $\sigma_{p}$ at any given level of $E_{p}$ ) which is impossible to beat. Although with the locations used in this research it was not possible to combine them efficiently without negative weights of at least one of them (which is a trait of nature of wind statistics at them), this absolute efficiency border is a good orientation regarding the portfolios that actually can be achieved.

As regards the problem with non-Gaussian character of wind generation, we carried out portfolio optimisation with four different goal parameters: ordinary standard deviation of the portfolio $\left(\sigma_{p}\right)$, left semi-deviation $\left(\sigma_{l}\right)$, right semi-deviation $\left(\sigma_{r}\right)$, and skewness $(S)$. Left semi-deviation is a square root of average square of all negative deviations of generation, while right semi-deviation is a square root of average square of all positive ones. Skewness is simply $\left(\sigma_{r} / \sigma_{l}\right)^{2}$. The solutions were then compared to assess the appropriateness of portfolio theory approach to the minimisation of wind generation variability.

As the second constraint brings along mathematical difficulties (that is, application of Kuhn-Tucker conditions instead of plain Lagrange multipliers), a numerical approach to solving optimisation problem was used. The portfolios were randomly generated by repeating random assignments of weight factors to the nine plant locations one million times. The population of portfolios obtained in that way was then searched for the best solutions for a given value of $E_{p}$ (that is, within a narrow strip of $0.99 E_{p}<E_{p}<1.01 E_{p}$ ). By "the best" we understood a solution with the lowest $\sigma_{p}, \sigma_{l}, \sigma_{r}$, or $S$, depending on which parameter was to be minimised. Sometimes, a subset of one hundred thousand portfolios was used for purposes of comparison of results obtained by different number of 
stochastically generated portfolios. The random generation of weight factors was performed like this: a sequence of $N-1$ random numbers were drawn from an interval $[0,1]$, using a uniform distribution. They were then ordered by ascending value. Then the interval $[0,1]$ was divided into $N$ sub-intervals delimited with that points. The length between each pair of adjacent points was then assigned as weight factor to a respective location. Such randomisation strategy was proven to generate diversely valued weight factors, which was good from the computational efficiency point of view.

The nine locations used for study will be kept in anonymity, as their identity is not important for the analyses. However, Tables 1-3 list their basic statistical properties.

In the continuation of this article the results will be displayed in the briefest possible form. Then the discussions on contributions, as well as conclusions, will be given.

Table 1. Basic statistics of energy production in nine Croatian wind plant locations during 2014 (source: authors' calculation)

\begin{tabular}{cccccccccc}
\hline & Loc. 1 & Loc. 2 & Loc. 3 & Loc. 4 & Loc. 5 & Loc. 6 & Loc. 7 & Loc. 8 & Loc. 9 \\
\hline$E[\%]$ & 32.7804 & 19.8216 & 30.1873 & 14.1026 & 21.0105 & 28.7657 & 21.1031 & 28.5285 & 26.8451 \\
$\sigma_{p}[\%$ points $]$ & 33.8036 & 24.4705 & 34.8772 & 21.6247 & 29.4103 & 33.0175 & 27.6059 & 31.1503 & 29.6501 \\
$\sigma_{l}[\%$ points $]$ & 26.0323 & 16.4927 & 25.1501 & 11.7777 & 17.0633 & 23.1354 & 17.2803 & 23.0299 & 22.8466 \\
$\sigma_{r}[\%$ points $]$ & 43.2632 & 35.0594 & 47.1265 & 36.5015 & 47.1673 & 45.7594 & 41.5016 & 40.9118 & 37.6786 \\
$S$ & 2.7619 & 4.5188 & 3.5112 & 9.605 & 7.6411 & 3.9121 & 5.768 & 3.1558 & 2.7199 \\
\hline
\end{tabular}

Table 2. Correlation matrix of the parameters from Table 1 (source: authors' calculation)

\begin{tabular}{cccccc}
\hline & $E$ & $\sigma_{p}$ & $\sigma_{l}$ & $\sigma_{r}$ & $S$ \\
\hline$E$ & 1 & 0.94359 & 0.99521 & 0.52193 & -0.87475 \\
$\sigma_{p}$ & & 1 & 0.93682 & 0.76709 & -0.72015 \\
$\sigma_{l}$ & & & 1 & 0.49542 & -0.89332 \\
$\sigma_{r}$ & & & & 1 & -0.13616 \\
$S$ & & & & & 1 \\
\hline
\end{tabular}

Table 3. Correlation matrix of 15 minute resolution time series of energy production at nine locations in Croatia in 2014 (source: authors' calculation)

\begin{tabular}{lccccccccc}
\hline & Loc. 1 & Loc. 2 & Loc. 3 & Loc. 4 & Loc. 5 & Loc. 6 & Loc. 7 & Loc. 8 & Loc. 9 \\
\hline Loc. 1 & 1 & 0.6755 & 0.587 & 0.6575 & 0.4849 & 0.7234 & 0.5422 & 0.7529 & 0.3849 \\
Loc. 2 & & 1 & 0.7097 & 0.6459 & 0.586 & 0.8632 & 0.7601 & 0.6077 & 0.1714 \\
Loc. 3 & & & 1 & 0.673 & 0.7161 & 0.7735 & 0.7475 & 0.6569 & 0.0775 \\
Loc. 4 & & & & 1 & 0.6733 & 0.6823 & 0.5937 & 0.7064 & 0.1436 \\
Loc. 5 & & & & & 1 & 0.6623 & 0.6248 & 0.6178 & 0.0965 \\
Loc. 6 & & & & & & 1 & 0.7964 & 0.6644 & 0.1892 \\
Loc. 7 & & & & & & & 1 & 0.5481 & 0.0582 \\
Loc. 8 & & & & & & & & 1 & 0.2654 \\
Loc. 9 & & & & & & & & & \\
\hline
\end{tabular}

\section{RESULTS}

Figure 1 displays a sample of twenty thousand randomly generated portfolios of the aforementioned nine wind plant locations. In reality, a total of one million portfolios was generated, but for the sake of picture's clarity, only every fiftieth was plotted. The population illustrated by this graph was searched to find portfolios that are optimal by various criteria, in a procedure explained above. The resulting efficient frontiers, one obtained from a subset of 100,000 portfolios, and the other from the whole one million ones, are plotted on Figure 2 together with an analytically calculated Markowitz's efficient frontier, the one obtained by solving the optimisation problem without non-negativity constraints on $r_{i}$ values. While the non-constrained curve is smooth, the 
constrained efficient borders are step-wise functions because they were obtained by approximate numerical procedure. If the number of randomly generated portfolios was much higher, say hundreds of millions, or billions, the width of the strip around target $E_{p}$ values could be proportionally narrower to obtain on average the same number of portfolios to compare within a strip, and consequently, the constrained efficient frontier would tend to become smooth, too. However, this additional computational complication would not bring any new information of value into our analysis. As one can see from Figure 2, increasing total number of random portfolios by a factor of ten contributed only slightly to more accurate estimation of efficient frontier.

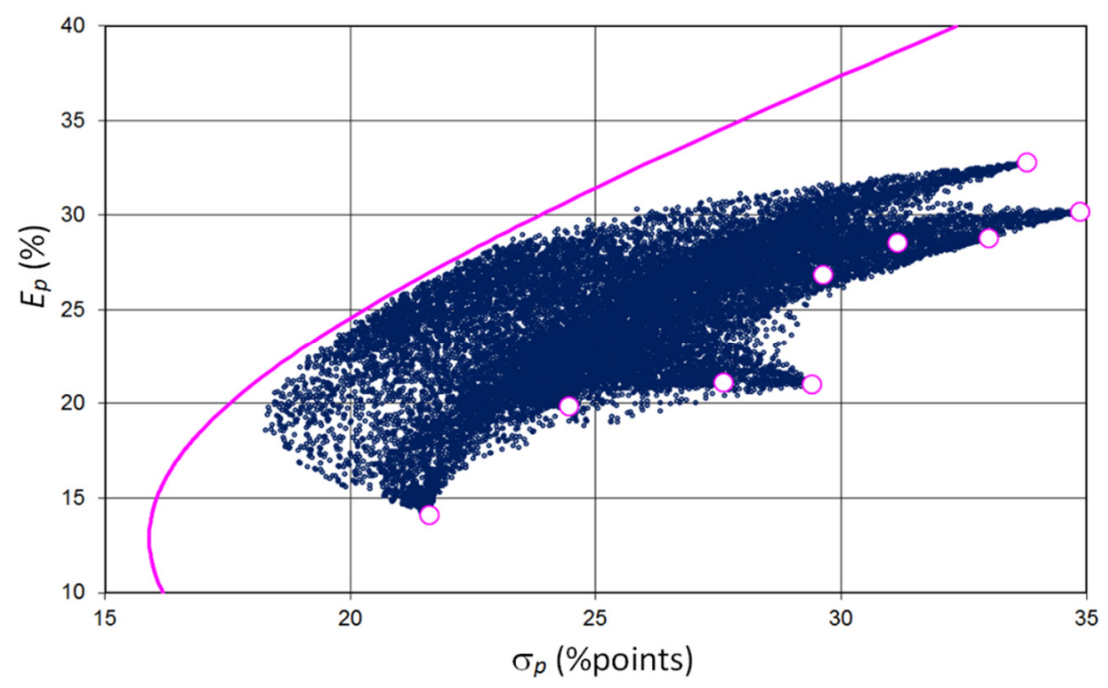

Figure 1. A sample of randomly generated portfolios, total number of program runs: 1 million, number of plotted portfolios: 20 thousand, small dots: random portfolios, large dots: nine individual locations, solid line: unconstrained efficient frontier

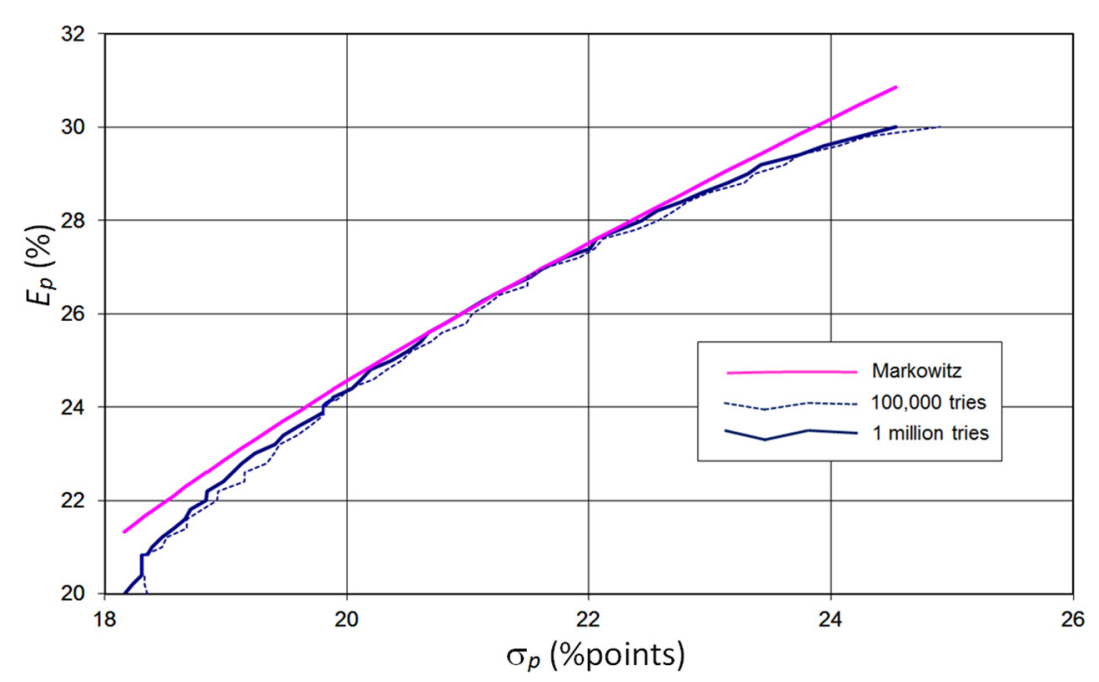

Figure 2. Efficient frontiers with and without ("Markowitz") non-negativity constraints, and with different size of randomly generated portfolio population

\section{Stability of algorithm}

A very important issue to tackle is stability of numerical estimation of efficient portfolios. Namely, if there were no non-negativity constraints, the relation between any of the $r_{i}$ 's and the $E_{p}$ would be linear, as it can be seen from the third constraint of the above stated optimisation problem, or from Figure 3, where analytically calculated values are displayed. Clearly, in non-constrained case the $r_{i}$ values change ideally 
smoothly along the efficiency border. However, in our case, where we must forbid negative values, the resulting weight factors of efficient portfolios cannot change linearly, anymore. However, none of the $r_{i}$ values should change too much, especially not in an abrupt way, when one moves along the efficient frontier from one efficient portfolio to a near another one. Note that in the presented example it is not possible to achieve unconstrained efficiency without at least one negative weight factor. This means that constrained efficiency frontier assumes strictly larger $\sigma_{p}$ values for any given $E_{p}$ than the non-constrained one. Figure 4 gives an example of the course of $r_{i}$ values in the vicinity of $E_{p}=25 \%$. Note that all the transitions are smooth, indicating that the "process of portfolio selection is stable" even with relatively coarse resolution along the efficient border (that is, relatively wide strips) for portfolio search. That would improve still more with growing number of random portfolios.

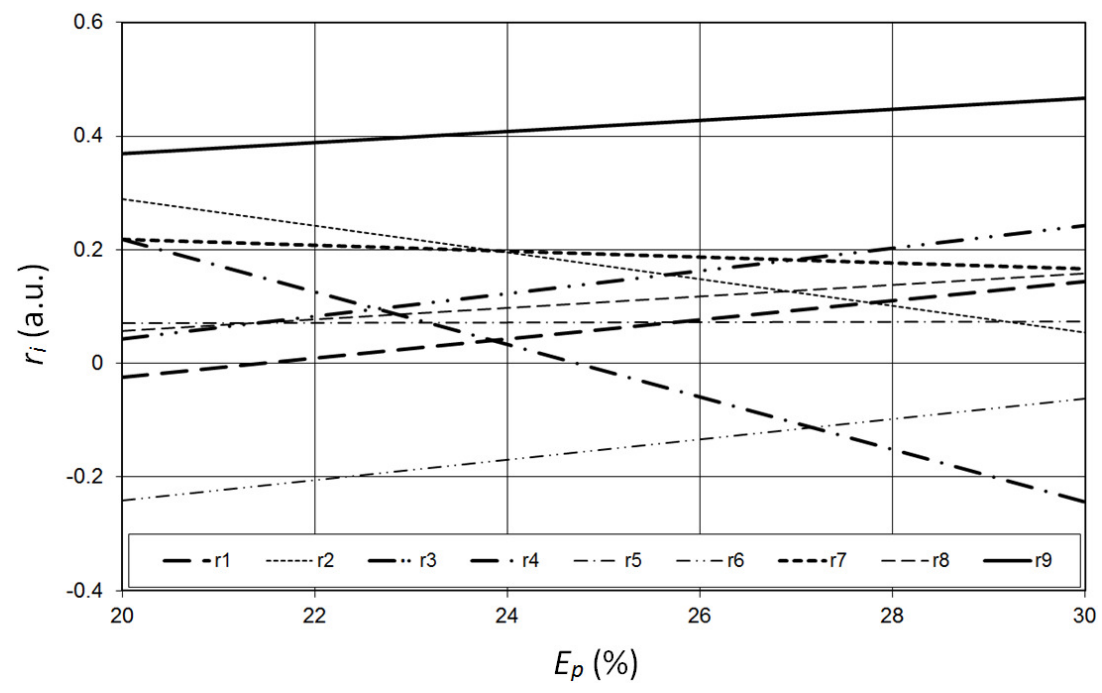

Figure 3. Weight factors of nine locations resulting from a non-constrained optimisation, note that there are no portfolios without at least one $r_{i}$ negative

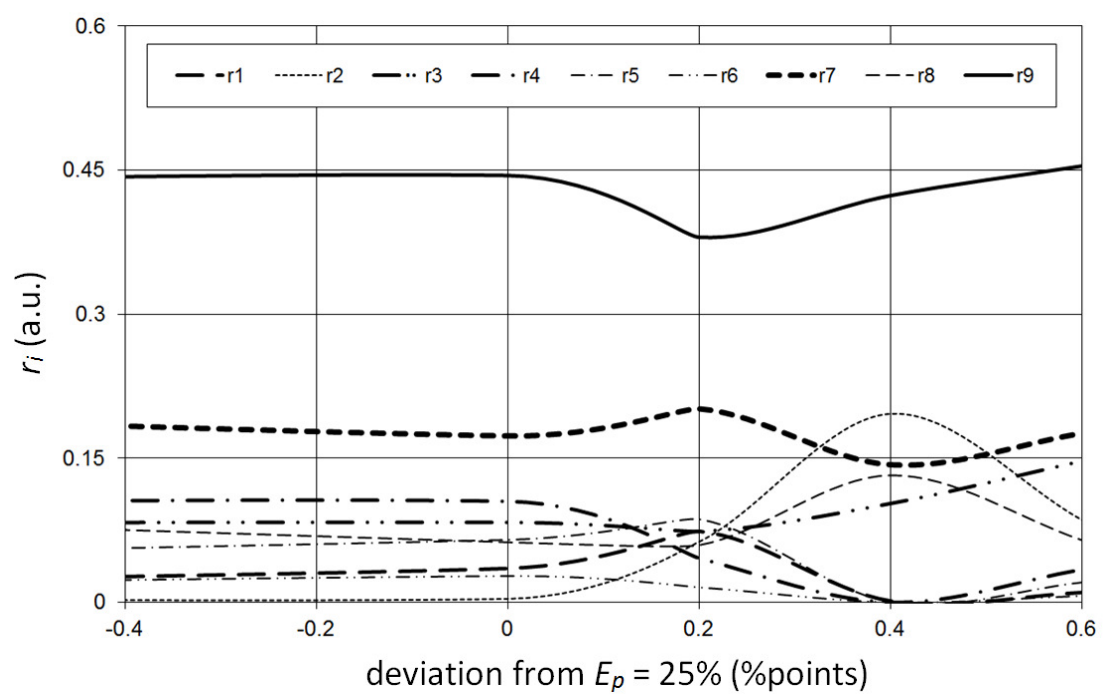

Figure 4. Weight factors of nine locations resulting from a constrained optimisation in the vicinity of $E_{p}=25 \%$, note there is no abrupt changes in any of the $r_{i}^{\prime}$ 's

\section{Participation of individual locations in a mix}

An interesting feature of optimisation for minimum variability is that a process of finding most efficient portfolios itself selects individual plant locations that are present 
with high weights in almost all portfolios. See for example $r_{9}$ or $r_{7}$ on Figure 3. On the other hand, there are locations that contribute only minutely to any of the efficient portfolios, like $r_{5}$. In the constrained case the "blocks" of "good" and "bad" locations are even more distinctive, because, naturally, the non-negativity constraint pushes at least the locations which assume negative values in non-constrained optimisation pretty close to zero. One way to quantify this phenomenon is to define "average participation" of each location in efficient portfolios, mean values of weight factors $r_{i}$ over the entire observed range of $E_{p}$ values. Table 4 gives the average participation across the set of nine locations, as well as standard deviations of their respective weight factors. Then, the correlation coefficient between the two nine-component row vectors were also calculated. As a rule of thumb, "the higher the $i$-th location's average participation, the lower the spread of its $r_{i}$ values". Thus, there are some locations that, "in the context of all observed ones", are considerably "better" than the others (here, especially Loc. 9 and 7), and there are some that are notably "worse" (here, especially Loc. 6 and 5). It should, however, be stressed that this is a consequence of "both" individual "and" group statistical behaviour of wind at the locations in question. In some other group's context the situation regarding any of the individual plant locations could in principle turn out to be different. To conclude, the portfolio selection algorithm "produces information on location quality", within the context of all available locations.

Table 4. Average participations of wind plant locations, and their standard deviations (expressed as percentages relative to the former), in portfolios along the efficient frontiers derived by minimisation of $\sigma_{p}$ (source: authors' calculation)

\begin{tabular}{cccccccccccc}
\hline & & Loc. 1 & Loc. 2 & Loc. 3 & Loc. 4 & Loc. 5 & Loc. 6 & Loc. 7 & Loc. 8 & Loc. 9 & Corr. ${ }^{*}$ \\
\hline \multirow{2}{*}{ No constraints } & Av. part. & 0.0598 & 0.1718 & 0.1428 & -0.013 & 0.0722 & -0.152 & 0.1923 & 0.1077 & 0.4177 & $-0.457^{*}$ \\
& St. dev. & 84.310 & 40.963 & 41.852 & $-1,074.0$ & 1.2132 & -35.39 & 8.0767 & 28.120 & 6.9949 & \\
Constraints $r_{i} \geq 0$ & Av. part. & 0.0605 & 0.0425 & 0.1186 & 0.1173 & 0.0368 & 0.011 & 0.1352 & 0.0627 & 0.4156 & -0.889 \\
& St. dev. & 155.2 & 119.61 & 99.399 & 118.15 & 110.38 & 155.08 & 71.983 & 99.906 & 12.152 & \\
\hline & "Correlation between average participations and their respective standard deviations \\
*This correlation coefficient was derived from averages and deviations of absolute values of $r$ ' $r_{i}$ 's, for otherwise it would not be comparable to the other \\
figure. However, the averages and deviations listed in the two rows left from the *-marked cell were calculated from true values of $r_{i}$ 's
\end{tabular}

\section{How non-Gaussian nature of wind generation affects optimisation?}

The next question to address, as it comes to application of portfolio optimisation theory to wind plants, pertains to the fact that distributions of energy produced by a system of wind plants are notoriously non-Gaussian (Sabolić [18]). Markowitz's theory, on the other hand, relies on an assumption of normal distribution of data. It is important to stress that this fact itself is not important if we use a numerical (or analytical, if we can) method to minimise some other parameter, rather than "plain" deviation $\sigma_{p}$. However, the question is whether the standard Markowitz's theory applies in case of skewed distribution type encountered in wind generation. To investigate this, we compared standard deviations of optimal portfolios found by searching for the ones with various parameters minimised. Ideally, there should be no difference, at all, because if the minimisation criteria were not important, minimisation of any of them would lead to exactly the same solutions, with exactly the same standard deviations. Consider Figure 5, where outcomes of optimisations by different criteria are illustrated. Let $\sigma_{p}{ }^{*}$ be standard deviation of a portfolio that has some of the other three parameters minimised, rather than "normal" $\sigma_{p}$. In the ideal case, all the curves would be constant and equal to 1 . One can see on the graph that minimisation by skewness leads to almost the same results as the one by standard deviation. Next, portfolios optimal by criterion of minimal right semi-variance are also very near (sometimes not different, at all) to the ones with minimised standard deviation, $\sigma_{p}$. However, minimisation of left semi-variance gives somewhat more notable differences in resulting portfolios. 


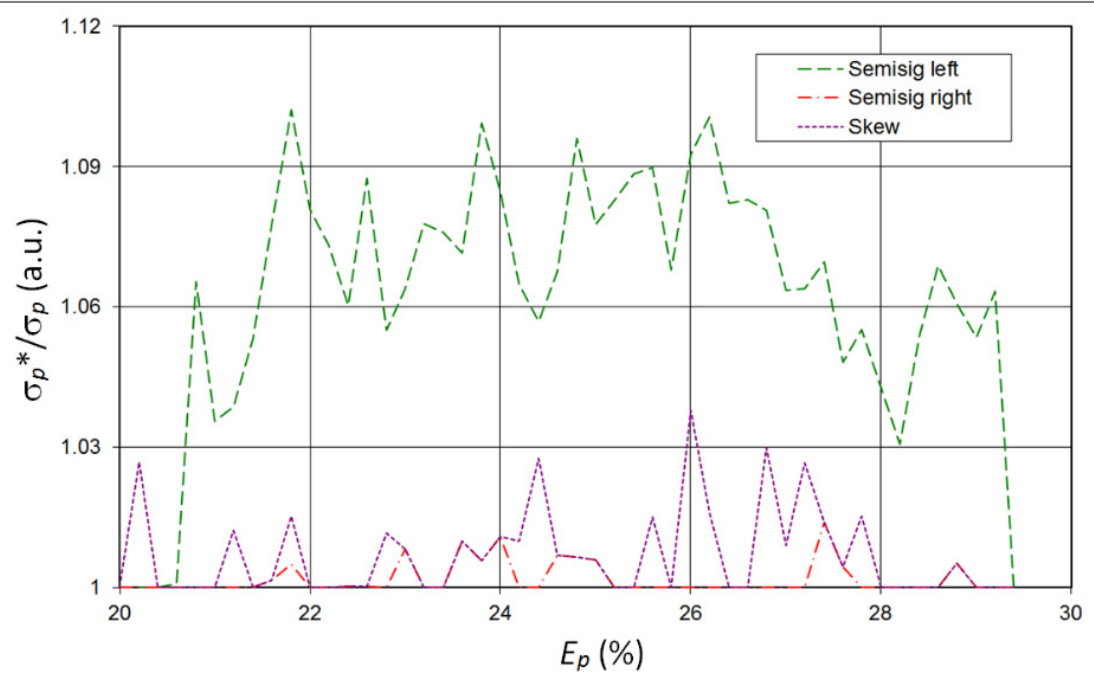

Figure 5. Ratio $\sigma_{p}{ }^{*} / \sigma_{p}$ along the efficiency frontier for different optimisation criteria used, $\sigma_{p}$ is standard deviation of portfolio with minimal $\sigma_{p}$, while $\sigma_{p}{ }^{*}$ is standard deviation of portfolio with minimal $\sigma_{l}$ (semisig left), $\sigma_{r}$ (semisig right), or $S$ (skew)

To assess by what degree the actual solutions for optimal portfolios differ, one can devise several methods. Here, the following one will be applied: Take the solution $r^{*}=\left[r_{1}{ }^{*} r_{2}{ }^{*} \ldots r_{N}{ }^{*}\right]$, obtained by minimisation of one of the parameters $\sigma_{r}, \sigma_{l}$, or $S$. Then, take the solution obtained by minimisation of "plain" standard deviation: $r=\left[\begin{array}{lll}r_{1} & r_{2} \ldots & \ldots\end{array}\right]$. Compute the following quantity: $M P D=(1 / N) \Sigma_{i}\left|r_{i}{ }^{*}-r_{i}\right|$, where summation goes from $i=1$ to $N$. Here, MPD stands for Mean Portfolio Distance. It measures how far actual solutions are from each other (that is, what is the average absolute difference of a weight factor obtained by minimisation of one of the three alternative parameters from the one obtained by minimisation of standard deviation). The ray chart for nine locations observed in this research is given on Figure 6. Note that optimisations by either skewness, or right semi-variance, give solutions very close to those coming out of the $\sigma_{p}$ minimisation. The left semi-variance optimisation again leads to somewhat bigger average differences in $r$ factors. Yet, as the maximum possible difference equals 1 , they are generally not too big.

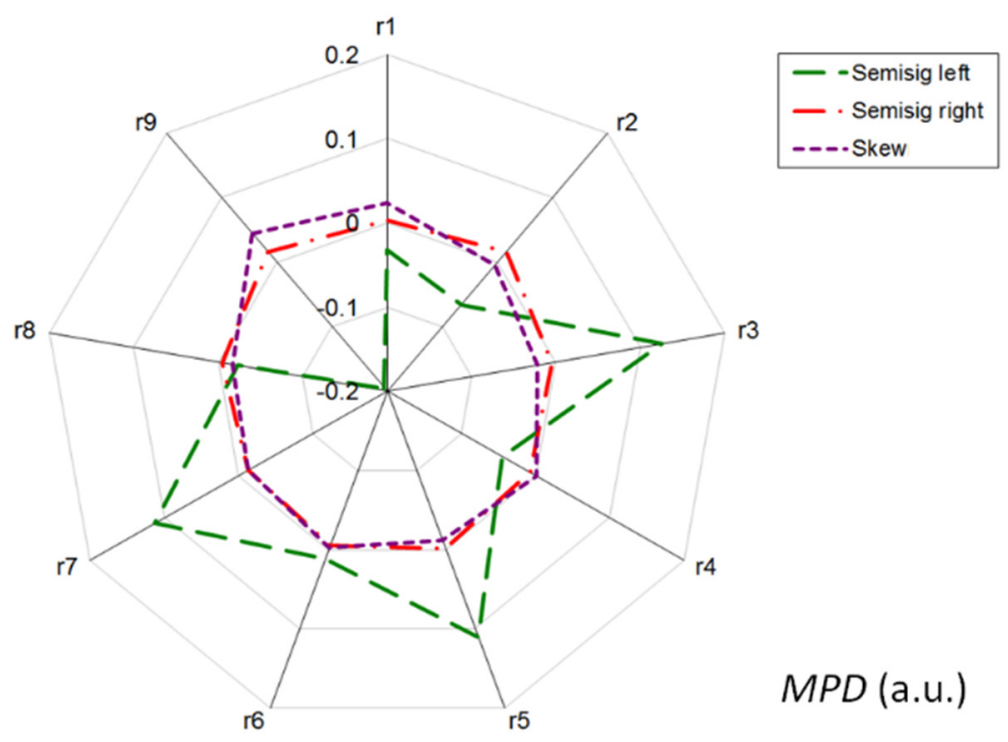

Figure 6. Mean portfolio distances for various minimisation criteria: semisig left $\left(\sigma_{l}\right)$, semisig right $\left(\sigma_{r}\right)$, skew $(S)$ 
Table 5 shows correlations between standard deviations of portfolios optimised by any of the aforementioned criteria, including the "ordinary" minimisation of variance without non-negativity constraints imposed. Note quite high values, although a bit lower when $\sigma_{l}$ is involved. These results suggest that "optimisation by any of the criteria yields similar results".

Table 5. Correlation matrix between strings of $\sigma$ values of efficient portfolios obtained by minimisation of different parameters $\left(\sigma_{p}, \sigma_{l}, \sigma_{r}\right.$, and $\left.S\right)$, for $E_{p}$ between 20 and 30 (source: authors' calculation)

\begin{tabular}{cccccc}
\hline Optimisation criterion & $\sigma_{p}$, Markowitz & $\sigma_{p}$ & $\sigma_{l}$ & $\sigma_{r}$ & $S$ \\
\hline$\sigma_{p}$, Markowitz & 1 & 0.9942 & 0.9708 & 0.9939 & 0.9945 \\
$\sigma_{p}$ & & 1 & 0.9706 & 0.9997 & 0.9960 \\
$\sigma_{l}$ & & & 1 & 0.9709 & 0.9659 \\
$\sigma_{r}$ & & & 1 & 0.9956 \\
$S$ & & & & & 1 \\
\hline
\end{tabular}

The solutions were found using analytical algorithm in no-constraint case ( $\sigma_{p}$, Markowitz), or numerically in constrained cases $\left(\sigma_{p}, \sigma_{l}, \sigma_{r}, S\right)$

\section{An example: Outcome of Croatian policy}

Table 6 enables one to compare an outcome of Croatian policy regarding the nine locations observed in this article to what would be optimal allocation of capacity between them for minimised variance. Croatian authorities did not have any particular strategy regarding capacity allocations, so that the policy outcome may be safely deemed random. Random decision may lead to any of the outcomes within the feasibility set visible on Figure 1. In the example studied here, the allocation yielded portfolio's standard deviation about ten percent worse than optimal. It does not look too much, but still, the cost of regulation is about ten percent higher than it could have been. This is a cost of pure managerial inefficiency as there is no other principle reason for it. The financial resources dissipated in such a way could have been used, for example, to regulate more wind plants, or to do anything else useful. In Table 6 a comparison between mean-variance ratios is given, too. This ratio, $E_{p} / \sigma_{p}^{2}$, is often used as a measure of satisfaction with the portfolio. By that criterion, Croatian policy outcome turned out to be "slightly worse than the optimal solution", but it is also slightly better than "centre of gravity" of feasibility set (which was estimated by calculating mean values of $E_{p}$, and $\sigma_{p}$, of all randomly generated portfolios from Figure 1). The latter is only relevant as a benchmark for expected outcome of random portfolio selection.

Table 6. Comparison of the outcome of Croatian policy regarding the nine locations observed in this research, and the optimal solution at $E_{p}=25.6 \%$, which is actual portfolio's measured load factor (source: authors' calculation)

\begin{tabular}{ccccccccccccc}
\hline & Loc. 1 $r_{1}$ & Loc. 2 $r_{2}$ & Loc. 3 $r_{3}$ & Loc. 4 $r_{4}$ & Loc. 5 $r_{5}$ & Loc. 6 $r_{6}$ & Loc. 7 $r_{7}$ & Loc. 8 $r_{8}$ & Loc. 9 $r_{9}$ & $E_{p}$ & $\sigma_{p}$ & $E_{p} / \sigma_{p}^{2}$ \\
\hline $\begin{array}{c}\text { Actual relative } \\
\text { shares in total } \\
\begin{array}{c}\text { capacity } \\
\text { Optimal shares }\end{array}\end{array}$ & 0.139 & 0.038 & 0.113 & 0.066 & 0.129 & 0.042 & 0.163 & 0.151 & 0.159 & 25.622 .8 & 0.049 \\
for the same $E_{p}$ & 0.010 & 0.086 & 0.147 & 0.034 & 0.021 & 0.007 & 0.176 & 0.065 & 0.454 & 25.620 .7 & 0.060 \\
& More data for comparison: & $\begin{array}{c}\text { Standard deviation is worse than optimum by 10.1\% } \\
\text { Mean-variance ratio for ,,centre of gravity“ of random portfolios: }\end{array}$ & 0.046 \\
\hline
\end{tabular}

\section{Influence of temporal resolution of data}

The next issue to address is how the optimal solution changes if one uses more coarse time series of data to extract statistical properties (means, variances, and co-variances) of wind plant locations. The original 15 minute time series for 2014 were converted to 
60 minute ones simply by summing each four consequent data points. Then, to compare outcomes, the non-constrained efficient frontiers were calculated for both data sets. Figure 7 shows the two, while Figure 8 displays error of $\sigma_{p}$ of an efficient portfolio. As one can see on both pictures, using rougher time resolution leads to a bit too optimistic results, meaning that the standard deviation of any efficient portfolio would be slightly underestimated. "In reality, deviation, and associated regulation costs, would be slightly higher than what optimisation results would suggest. However, the error is rather minute", and given other possible sources of imprecision, it seems not to have too much practical significance. Naturally, it is always better to use finer data if one has them available.

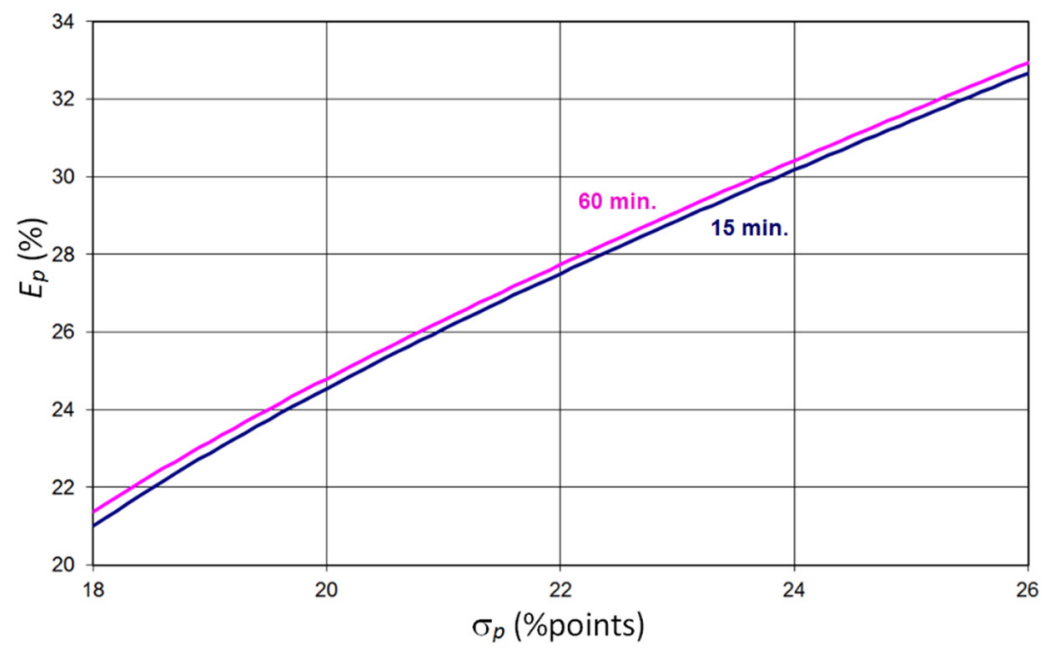

Figure 7. Comparison of unconstrained efficient frontiers calculated for the same nine wind plant locations, based on their 2014 data, with 15 minute or 60 minute time resolution

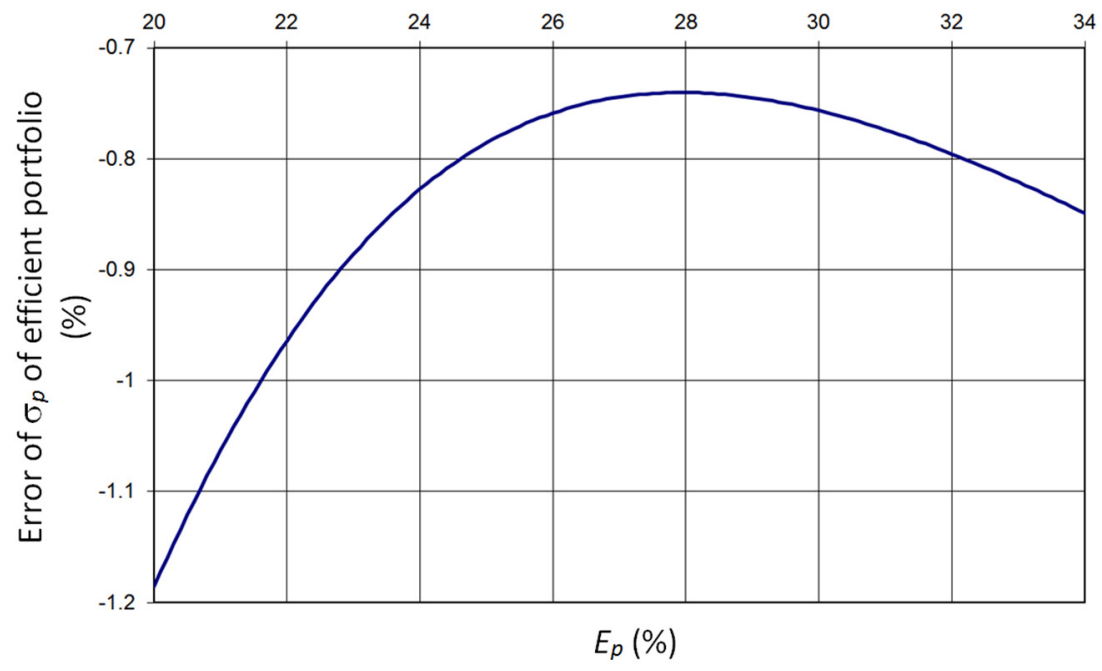

Figure 8. Error of $\sigma_{p}$ estimated from 60 minute time series, relative to the one computed from 15 minute ones

\section{Optimal upgrading of a system with already operating variable sources}

Finally, one can ask what if there is already a set of already operating wind plants, and there are plans to build more. Usually, a policy maker cannot deprive owners of existing plants of the rights granted to them in the past. They must be taken into account as given. The set of existing plants as a whole has historically measured data on energy production, probably in 15 minute resolution. So, the total installed power, average production, and deviations from average, are known. If the policy maker had data of wind speeds at new 
locations in the same roster, he could easily "optimise allocation of intended new capacity between new locations by taking the pre-existing situation as just another constraint" in problem formulation, that is:

$$
r_{0}=C_{0} /\left(C_{0}+C\right)
$$

where $r_{0}$ is the weight factor assigned to the set of pre-existing plants, $C_{0}$ is the total installed capacity of the same set, and $C$ is new capacity to be assigned to the new locations. Now, in the problem formulation (1), all summations must run from 0 to $N$ (instead from 1 to $N$, as before).

\section{CONCLUSIONS AND FUTURE RESEARCH}

The research described in this article led to the following contributions:

- Modern portfolio theory can be successfully used as a conceptual tool for deciding on optimal allocation of new generation capacity to be installed at new plant locations with a goal of minimisation of generation variability;

- Application of the basic form of portfolio theory (that is, minimisation of "plain" standard deviation in spite of the non-Gaussian nature of wind plant energy output) will lead to only slightly sub-optimal solution;

- Using right semi-variance, or the distribution skewness, as goal functions to be minimised gives practically the same solutions as minimisation of portfolio's standard deviation;

- Statistical reduction of variability is limited in nature, and it should be utilised as much as possible, hence the need to perform optimisation as the one described here;

- Blindfold allocation of new capacities to be installed among several new locations leads by necessity to inefficiency, and thus to unnecessarily increased costs of regulation of generation variability. Policy measures aiming at elimination of costs that can be avoided by organisational measures are, naturally, socially useful and desirable, but they provide benefits to the industry, too, as avoiding unnecessary costs releases resources for other more useful purposes.

It should be stressed that this work deals with minimisation of one single variable - be it standard deviation, or some other measure of production variability. An obvious direction for further research is introduction of other possible components into the goal function, because there may as well be other costs related to locations of wind plants. However, such a multidimensional analysis was outside the intended scope of our research.

\section{REFERENCES}

1. Roques, F., Hiroux, C. and Saguan, M., Optimal Wind Power deployment in Europe A Portfolio approach, Energy Policy, Vol. 38, No. 7, pp 3245-3256, 2010, https://doi.org/10.1016/j.enpol.2009.07.048

2. Katzenstein, W., Fertig, E. and Apt, J., The Variability of interconnected Wind Plants, Energy Policy, Vol. 38, No. 8, pp 4400-4410, 2010, https://doi.org/10.1016/j.enpol.2010.03.069

3. Ueckerdt, F., Hirth, L., Luderer, G. and Edenhofer, O., System LCOE: What are the Costs of Variable Renewables?, http://papers.ssrn.com/sol3/papers.cfm?abstract_id=2200572, 2013, [Accessed: 27-August-2016]

4. Gross, R., Heptonstall, P., Anderson, D., Green, T., Leach, M. and Skea, J., The Cost and Impacts of Intermittency: An assessment of the Evidence on the Costs and Impacts of Intermittent Generation on the British Electricity Network, 2006, 
http://www.ukerc.ac.uk/asset/BCD3C9C4-FCC1-4DAD-896A95960CD61D51/, [Accessed: 23-November-2016]

5. Milligan, M. and Kirby, B., Calculating Wind Integration Costs: Separating Wind Energy Value from Integration Cost Impacts, NREL Technical Report TP-550-46275, http://www.consultkirby.com/files/NREL-TP-550-46275_Calculating_Wind_Inegratio n_Costs.pdf, 2009, [Accessed: 23-November-2016]

6. Smith, C., Milligan, M., DeMeo, E. and Parsons, B., Utility Wind Integration and Operating Impact State of the Art, IEEE Trans. Power Syst., Vol. 22, No. 3, pp 900-908, 2007, https://doi.org/10.1109/TPWRS.2007.901598

7. Holttinen, H., Melbom, P., Orths, A., Lange, B., O’Malley, M., Tande, J. O., Estanqueiro, A., Gomez, E., Söder, L., Strbac, G., Smith, J. C. and van Hulle, F., Impacts of Large Amonuts of Wind Power on Design and Operation of Power Systems, Results of IEA Collaboration, Wind Energy, Vol. 14, No. 2, pp 179-192, 2011, https://doi.org/10.1002/we.410

8. DeCesaro, J. and Porter, K., Wind Energy and Power System Operations: A Review of Wind Integration Studies to Date, NREL Subcontract Report SR-550-47256, 2009, http://www.nrel.gov/docs/fy10osti/47256.pdf, [Accessed: 23-November-2016]

9. GE Energy, Western Wind and Solar Integration Study, NREL Subcontract Report SR-550-47434, http://www.nrel.gov/docs/fy10osti/47434.pdf, 2010, [Accessed: 23-November-2016]

10. Fripp, M. and Wiser, R. H., Effects of Temporal Wind Patterns in the Value of Wind-generated Electricity in California and the Northwest, IEEE Trans. Power Syst., Vol. 23, No. 2, pp 477-485, 2008, https://doi.org/10.1109/TPWRS.2008.919427

11. Borenstein, S., The Market Value and Cost of Solar Photovoltaic Electricity Production, $\begin{array}{llll}\text { CSEM Working 176, } & \text { Paper }\end{array}$ http://isites.harvard.edu/fs/docs/icb.topic541736.files/Borenstein2008.pdf, $\quad 2008$, [Accessed: 23-November-2016]

12. Lamont, A., Assessing the Long-term System Value of Intermittent Electric Generation Technologies, Energy Econ., Vol. 30, No. 3, pp 1208-1231, 2008, https://doi.org/10.1016/j.eneco.2007.02.007

13. Mills, A. and Wiser, R., Changes in the Economic Value of Variable Generation at High Penetrations Levels: A Pilot Case Study of California, 2012, https://emp.lbl.gov/sites/all/files/lbnl-5445e.pdf, [Accessed: 23-November-2016]

14. Nicolosi, M., The Economics of Renewable Electricity Market Integration, An Empirical and Model-based analysis of Regulatory Frameworks and their Impacts on the Power Market, University of Cologne, Koln, Germany, Ph.D. Thesis, http://kups.ub.uni-koeln.de/4612/, 2011, [Accessed: 23-November-2016]

15. Hirth, L., The Market Value of Variable Renewables: The Effect of Solar and Wind Power Variability on Their Relative Price, Energy Econ., Vol. 38, pp 218-236, 2013, https://doi.org/10.1016/j.eneco.2013.02.004

16. Markowitz, H., Portfolio Selection, The Journal of Finance, Vol. 7, pp 77-91, 1952, https://doi.org/10.1111/j.1540-6261.1952.tb01525.x

17. Elton, E. J. and Gruber, M. J., Modern Portfolio Theory, pp 2-9, 1950 to Date, Stern School of Business, New York University, New York, USA, 1997, http://archive.nyu.edu/bitstream/2451/26674/2/FIN-97-003.pdf, [Accessed: 27-August-2016]

18. Sabolić, D., Statistical Properties of Electricity Generation From a Large System of Wind Plants, and Demand For Fast Regulation, $2^{\text {nd }}$ South East European Conference on Sustainable Development of Energy, Water, and Environment Systems, Piran, Slovenia, June 15-18, 2016.

19. Monahan, A. H., He, Y., McFarlane, N. and Dai, A., The Probability Distribution of Land Surface Wind Speeds, Journal of Climate, Vol. 24, pp 3892-3909, 2011, https://doi.org/10.1175/2011JCLI4106.1 
20. Ouarda, T. B. M. J., Charron, C., Shin, J.-Y., Marpu, P. R., Al-Mandoos, A. H., Al-Tamimi, L. H., Ghedira, H. and Al Hosary, T. N., Probability Distributions of Wind Speed in UAR, Energy Conversion and Management, Vol. 93, pp 414-434, 2015, https://doi.org/10.1016/j.enconman.2015.01.036

21. Merton, R. C., An Analytic Derivation of the Efficient Portfolio Frontier, The Journal of Financial and Quantitative Analysis, Vol. 7, No. 4, pp 1851-1872, 1972, https://doi.org/10.2307/2329621

22. Lando, D. and Poulsen, R., Lecture Notes for the Course Investerings- og Finansierinsteori, pp 131-152, University of Copenhagen, Copehhagen, Denmark, 2001, http://www.math.ku.dk/ rolf/teaching/iff01/, [Accessed: 27-August-2016] 